\title{
Feminismos en torsión
}

\author{
Torsion Feminisms ${ }^{1}$
}

\begin{abstract}
MARIA MEDINA-VICENT (Universitat Jaume I), SONIA REVERTER-BAÑÓN (Universitat Jaume I) e IRENE STRAZZERI (Università degli studi di Foggia)
\end{abstract}

Medina-Vicent, Maria, Reverter-Bañón, Sonia y Strazzeri, Irene (2019). Feminismos en torsión. Recerca. Revista de Pensament i Análisi, 24(2), pp. 1-14.

\section{Resumen}

En un momento de gran efervescencia feminista, se entremezclan las reclamaciones políticas del movimiento con las peligrosas reapropiaciones que de sus núcleos de lucha hace el neoliberalismo. Las tensiones que se presentan en este contexto son muchas y variadas, algunas evidentes y otras invisibles, pero todas contribuyen a la torsión actual del feminismo. En el presente artículo se abordarán algunas de estas tensiones que nacen en la intersección entre feminismo y neoliberalismo: desde la publicidad como posible herramienta feminista hasta los discursos sobre el emprendimiento y el empleo, pasando por el diseño de políticas públicas, y la mercantilización de los cuerpos femeninos. También se abordarán algunas de las posibles rearticulaciones y respuestas políticas feministas ante esta realidad, mostrando la complejidad de los retos a los que tienen que hacer frente los feminismos hoy.

Palabras clave: feminismos, neoliberalismo, desigualdad, lucha política.

\section{Abstract}

In times of great feminist effervescence, the political claims of the movement are intermingled with the dangerous reappropriations that neoliberalism makes of its struggle nuclei. The tensions that appear in this context are many and varied, some obvious and some invisible, but all of them contribute to the torsion of feminism. This article will address some of the tensions that arise from the intersection between feminism and neoliberalism: from advertising as a possible feminist tool, to speeches on entrepreneurship and employment, through the design of public policies, and the commodification of female bodies. Some of the possible re-articulations and feminist political responses to this reality will be addressed, showing the great complexity of the challenges that feminisms face today.

Key Words: feminisms, neoliberalism, inequality, political struggle.

\footnotetext{
Este trabajo se enmarca en el Proyecto de Investigación Científica y Desarrollo Tecnológico FFI201676753-C2-2-P, financiado por el Ministerio Español de Economía y Competitividad y el Proyecto de Investigación Científica y Desarrollo Tecnológico del Plan de Promoción de la Investigación de la Universitat Jaume I para el año 2016 UJI-A2016-04.
} 


\section{INTRODUCCIÓN}

Las palabras feminismo y feminista son hoy parte irrenunciable de la agenda mediática. De la reticencia generalizada propia de hace unas décadas a autodenominar-se feminista, hemos pasado a encontrar lemas profeministas en ropa de tiendas low cost como Zara, Mango o Primark. Dicha popularización del feminismo (Banet-Weiser, 2018) representa una nueva etapa con características novedosas sobre las que cabe reflexionar. Pero no hay que olvidar que, más allá de esta creciente visibilidad, dicho concepto esconde siglos de lucha política por la igualdad entre mujeres y hombres (Reverter-Bañón, 2001, 2009). Poner en equilibrio ambas facetas del movimiento por la igualdad es una de las tareas del presente monográfico, donde se pretenden identificar los dilemas y retos - o tensiones, tal y como las hemos llamado- que se desprenden de la construcción de una subjetividad feminista en la cultura neoliberal y el establecimiento de una crítica radical de las estructuras sociales y materiales de la desigualdad en dicho contexto.

Antes de avanzar cabe recordar que el neoliberalismo no remite solamente a un sistema económico que promueve la desregulación del comercio, la privatización de servicios estatales y la reducción de gasto social, entre otras operaciones. Se refiere también a una dimensión subjetiva y disciplinaria que promueve la incorporación por parte de los sujetos de la lógica neoliberal (Laval y Dardort, 2018). Una subjetividad neoliberal basada en el emprendimiento, la autoexigencia y el individualismo, convirtiendo la propia vida en ámbito laboral (Gill, 2019). Esta subjetividad intersecciona también con el feminismo, reconceptualizando parte de su sentido (Scharff, 2016a, 2016b; Rottenberg, 2018a, 2018b; Medina-Vicent, 2018a, 2018b). Esta realidad debe enmarcarse también en la etapa postfeminista, donde vemos un feminismo reemplazado por un agresivo individualismo que proclama: antes que mujeres, somos individuos (Projansky, 2001; McRobbie, 2004, 2009; Tasker y Negra 2007; Gill, 2007).

Así pues, esta visión neoliberal del feminismo convive con una reactivación de la raíz política del mismo, lo que produce la torsión del movimiento abriendo paso a diferentes tensiones. La reacción feminista a las políticas racistas y machistas de Donald Trump mediante las Women's March celebradas en enero del 2017, 2018 y 2019 son un ejemplo de esta reactivación. No obstante, este cambio se viene gestando desde hace tiempo, siendo también el papel de las luchas feministas en Latinoamérica clave para la reemergencia del movimiento a nivel global (Schild, 2016). Por ejemplo, la emergencia de los gritos 
\#NiUnaMenos y \#VivasNosQueremos marcó el inicio de las huelgas feministas a nivel global desde Argentina, siendo también España uno de los epicentros de dichas reclamaciones. Además, en los últimos años hemos sido testigos de campañas como el \#metoo, \#yosítecreo, los abanicos rojos que llenaron la gala de los Goya con el lema \#MásMujeres o el movimiento \#Time'sUp (Maseda García y Gómez Nicolau, 2018). Sin duda, estas son algunas de las campañas sociales, eminentemente desarrolladas mediante las redes sociales, que han ayudado a poner en el debate público temáticas que antes quedaban en la sombra, como por ejemplo el acoso sexual. Por esta razón, podemos preguntarnos acerca de las tensiones que se presentan ante el movimiento feminista en esta etapa marcada por la lógica neoliberal.

Así pues, este monográfico se inscribe en el campo de la teoría política feminista, que pretende reflexionar en torno a las posibilidades de establecer una crítica radical al poder desde las bases del feminismo. Es decir, encontrar las implicaciones políticas y sociales que tiene el actual auge del feminismo en los medios de masas, las redes sociales y la opinión pública, así como la existencia de un compromiso político real con la transformación social. Por tanto, incluye propuestas relacionadas con la teoría política feminista desde diferentes perspectivas, enfatizando aquellos temas relacionados con los discursos contemporáneos del feminismo dentro del neoliberalismo. Se abordarán cuestiones que van desde la publicidad como posible herramienta feminista hasta los discursos sobre el emprendimiento y el empleo, pasando por el diseño de políticas públicas y la mercantilización de los cuerpos femeninos.

\section{LAS TENSIONES DEL FEMINISMO EN EL MARCO NEOLIBERAL}

Son muchas las tensiones que caracterizan al movimiento feminista en la etapa neoliberal, y es que, aunque a primera vista podríamos señalar que las bases de dicho sistema se contraponen con los principios del feminismo, esto no imposibilita que se esté produciendo una confluencia de ambos, teniendo como consecuencia la transformación del feminismo en algo que difiere de forma notable de su historia de lucha por la igualdad, pero también la libertad. Esto demuestra la urgencia de aproximarse críticamente a los núcleos de torsión del feminismo por parte del neoliberalismo. Así pues, este número monográfico trata de acercarse a algunas de dichas tensiones, dejando la puerta abierta al planteamiento y la reflexión de muchas otras distintas formas en que la lógica neoliberal se diluye en los principios feministas. 
En este sentido, M. ${ }^{a}$ Isabel Menéndez establece una interesante reflexión sobre los potenciales y los riesgos de la incorporación de los discursos feministas a la comunicación publicitaria, señalando el necesario paso de la llamada Femvertising a la Publicidad Profem. Evidentemente, al hablar de la intersección entre movimiento feminista y publicidad, surge la duda de la posible instrumentalización del feminismo en función de objetivos comerciales. Aquí encontramos una de las primeras tensiones analizadas en este monográfico. Y es que el carácter persuasivo de la publicidad unido al continuo recurso a utilizar estereotipos (de todo tipo) hacen que sea difícil entrever las posibilidades de la publicidad como una herramienta de empoderamiento para las mujeres.

Siendo escasa la bibliografía especializada sobre Femvertising, el trabajo de Menéndez contribuye a trazar un recorrido desde los orígenes de la misma, apuntando los nudos conflictivos que deben abordarse desde la teoría feminista. Entre estos se encuentra la tendencia actual de la publicidad de aunar activismo y feminismo a través del consumo, una apropiación del feminismo con fines comerciales. Al reducir la lucha feminista a un producto de consumo, se mercantiliza su lucha (Gill, 2008), reforzando las reticencias a pensarse colectivamente (Menéndez, 2015, 2017). No obstante, Menéndez sí afirma la posibilidad de pensar publicidad que genere feminismo proponiendo el nombre de Advertising Profem o Publicidad Profem, es decir, construir mensajes publicitarios capaces de incitar al público a la reflexión y la acción feministas, promoviendo el cambio social.

Sin duda, las formas en que se entremezclan los discursos neoliberales con los feministas son diversas y, por tanto, otra de las tensiones presentes en el imaginario actual se refiere a cómo las instituciones públicas se nutren o responden a las exigencias neoliberales cuando se trata de promover el empleo femenino. Carlota Carretero García y Andy Eric Castillo Patton abordan el papel de las políticas e iniciativas públicas de promoción del empleo y el emprendimiento entre mujeres desarrolladas por el Ayuntamiento de Madrid (2015-2019), centrándose en los casos del Premio Emprendedoras y Juntas Emprendemos en Madrid. Este marco encontraría una interacción con determinadas ideas feministas vinculadas al empoderamiento económico, la capacidad de elección o la agencia individual enmarcadas en algunas versiones liberales del feminismo.

Sin duda, una de las claves de su análisis reside en la capacidad de estas iniciativas para generar ideales subjetivos de feminidad que reproducen las dicotomías de género y los ideales subjetivos femeninos, y cómo esto intersecciona con la lógica de la «empresa de uno/a mismo/a» y la constante inversión 
en capital humano (Muñoz-Rodríguez y Santos Ortega, 2017). Así, ambas iniciativas estarían atravesadas por lógicas neoliberales e ideas feministas, pero al mismo tiempo representarían dos casos dispares, el primero enmarcado en la lógica del feminismo neoliberal (Rottenberg, 2018a, 2018b; Medina-Vicent, 2018a), y el otro abierto a la formulación de otros modelos. Ambos programas coinciden en la promoción de las subjetividades neoliberales emprendedoras basadas en la autorresponsabilización y autogestión de sus proyectos y proyecciones. De este modo, una institución pública como es el Ayuntamiento de Madrid estaría contribuyendo a la profusión de discursos neoliberales del emprendimiento, reconceptualizando el desempleo como una cuestión que deben resolver los individuos (Medina-Vicent, 2018c; Serrano, Fernández Rodríguez y Artiaga, 2012).

En relación al papel de instituciones y Estados en la profusión de dichos discursos, Montserrat Crespín Morales analiza el contexto japonés, específicamente el concepto womenomics, referido a la adopción del bloque de medidas políticas por parte del actual primer ministro japonés Shinzō Abe, que propone la idea de «comprar la economía femenina» tratando de incluir a las mujeres en el mercado laboral para así corregir un futuro estancamiento del crecimiento económico del país. Crespín se encarga de discernir si el recurso a las mujeres trata de alcanzar la igualdad o solamente sirve para reforzar un patrón productivista, resignificando las aspiraciones feministas en beneficio del sistema neoliberal (Mackie, 2016).

La autora revela que dicho bloque de medidas permite llevar a cabo una de las operaciones centrales del neoliberalismo, esto es, la potenciación de la desregulación económica y de la normativa laboral. Este proceso, a diferencia de promover la igualdad, acaba por beneficiar solamente a las mujeres en una posición social alta, mientras que las demás quedan sumidas en la precariedad. Así, mediante un proceso de desregulación económica como el analizado por Crespín, que dice tener por objeto central resituar a las mujeres en el mundo del trabajo y reconocerlas como motor central del éxito del país, se esconden procesos de resignificación que perpetúan las desigualdades y las diferencias, acrecentando el empobrecimiento de las mujeres. Ahora sí, el responsable no sería tanto el Estado, antes garante de la vida de estas mujeres, si no ellas mismas, convertidas en responsables de su propio destino en un marco de feroz neoliberalización de la economía.

Dentro de este marco de incorporación de las mujeres al mercado, entre la lógica del emprendimiento y las empresarias de sí mismas, Alicia Valdés Lucas se plantea la posibilidad de que el capital erótico (Hakim, 2011) pueda 
ser considerado una herramienta feminista para la subversión de las jerarquías y relaciones económicas y pueda favorecer una liberación de las mujeres. Valdés apunta que la teoría de Hakim reproduce el sujeto homogéneo del feminismo referido a la mujer blanca, cisgénero y con recursos económicos. De este modo, la subjetividad a la que acceden las mujeres por medio del capital erótico es en clave de consumidoras: dichas mujeres deben contar con una posición económica ventajosa que les permita invertir tiempo y dinero en explotar su capital erótico (Moreno Pestaña, 2016). Así, el individualismo propio de un feminismo neoliberal centrado en la elección de consumo como virtud máxima de la igualdad y la libertad viene garantizado por una estructura que otorga seguridad y capacidad de decisión a dichas mujeres, algo que desvela el olvido de las diferentes variables que intervienen en la desigualdad de género.

Sin embargo, Valdés apunta acertadamente que el hecho de desarrollar una subjetividad empoderada como consumidoras no implica la adquisición de derechos y libertades que mejorarán la posición de las mujeres en una sociedad masculina y androcéntrica. Así, dicha autora sostiene que el feminismo blanco y liberal ha construido sus peticiones sin cuestionar los principios fundamentales de la economía liberal y que esto demuestra que la implementación del capital erótico no conduce a una subversión feminista de la economía, sino que fortalece el sistema económico y político liberal.

Por otro lado, al analizar las tensiones entre feminismo y neoliberalismo no se puede pasar por alto la creciente mercantilización de los cuerpos de las mujeres (Medina-Vicent, 2018d). La tensión entre el feminismo y la demanda de prostitución es analizada por Rosa M. Senent Julián, centrándose en un análisis del discurso online de los consumidores de prostitución. El debate feminista sobre la prostitución sigue abierto y en su seno podemos encontrar posiciones muy polarizadas. En este debate, la aportación de Senent se centra en reflexionar en torno al sistema de creencias de los sujetos prostituyentes (Ranea, 2017), para comprobar si las relaciones que establecen con las prostitutas están basadas en términos igualitarios o en la dicotomía dominaciónsubordinación característica de las relaciones de poder de género (Sambade, 2017).

De los análisis se desprende que la objetificación y la deshumanización de las mujeres es una constante en la concepción de los sujetos prostituyentes, algo que implica la perpetuación de la violencia contra las mujeres. Y es que, en la conversión del cuerpo femenino en mercancía sujeta a compraventa se legitima el poder económico de los hombres (De Miguel, 2015). Así pues, señala Senent, en el discurso neoliberal de la prostitución como un trabajo más 
intervienen grandes intereses económicos (Ekman, 2013), produciendo una cooptación deliberada y estratégica de los términos feministas (Strazzeri, 2019; Gimeno, 2018; Alario, 2018). Tal y como apunta Senent, las feministas deberían contraatacar la capacidad del capitalismo patriarcal de fagocitar el potencial revolucionario del movimiento, cuestionándose la legitimidad de los discursos neoliberales mientras responsabilizan a los hombres por la perpetuación de la prostitución. Deberían solicitar a los gobiernos que adoptaran un enfoque legal y sensible al género, que aborde la demanda y se comprometa verdaderamente a luchar contra la violencia contra las mujeres en sus diversas formas.

Tal y como se ha podido observar, en la torsión que el sistema neoliberal produce sobre el movimiento feminista, surgen diferentes tensiones que abren a su vez interesantes debates dentro de la teoría política feminista. Esto nos demuestra que debemos mostrarnos atentas a los virajes discursivos que se produzcan en el mismo. No obstante, también se vuelve urgente recuperar la raíz política del feminismo y rearticular una lucha a nivel global, una lucha que se base en una noción de cosmopolitismo feminista contra las nefastas consecuencias de la globalización (Reverter-Bañón, 2017). En el siguiente subapartado trataremos de poner en valor algunas de estas cuestiones.

\subsection{Rearticulaciones de la lucha feminista}

Tal y como se ha podido observar en el punto anterior, la necesaria reflexión crítica y feminista sobre las estructuras de perpetuación de la lógica neoliberal son más que necesarias hoy. Cómo articular dicha lucha es un debate aún en progreso que no cuenta con una sola respuesta. En este sentido, Marta Vaamonde Gamo explora la propuesta de Nancy Fraser (1997, 2000, 2011, 2015), que concibe la paridad en la participación con el objetivo de ampliar la justicia social en las sociedades neoliberales.

Dicho planteamiento permite abordar las distintas dimensiones de la justicia (económica, cultural y política), establecer un análisis multidimensional de los ámbitos de dominación de las mujeres y desarrollar una concepción que tiene en cuenta diferentes variables que interseccionan para generar la desigualdad. La interpretación de Fraser de la justicia como paridad participativa amplía e integra, desde su punto de vista, los aspectos más interesantes de otros planteamientos: supera el economicismo de los planteamientos marxistas y el culturalismo de las políticas de la identidad integrando sus luchas por la distribución y por el reconocimiento, a las que une las luchas por la represen- 
tación. De esta manera, su propuesta permite analizar mejor las complejas injusticias y articular medidas en pro de la justicia de género.

Sin embargo, Vaamonde plantea un límite a la propuesta de Fraser que es no analizar en profundidad los condicionantes subjetivos de los que también depende. Por eso sugiere superar esos límites ampliando el ideal normativo de Fraser con el pragmatismo de John Dewey, que tiene en cuenta los condicionantes subjetivos de la acción. Ambos coinciden en considerar la democracia como un ideal social que depende de la participación en condiciones de igualdad y pretenden su radicalización; pero Dewey proporciona un análisis funcional de las transacciones de las que la subjetividad y el orden social son factores. De esta manera, Dewey amplía la consideración normativa de la paridad en la participación, interpretándola no solo como un procedimiento de organización de una sociedad plural, sino como medio de crecimiento personal, como principio que nos permite construir una personalidad autónoma. Atiende así no solo a las reclamaciones sociales, a las luchas, imprescindibles para extender la paridad participativa, sino también a las actitudes personales que requiere y resultan imprescindibles para la extensión y radicalización democrática.

En este sentido, Pablo Pérez Navarro es el encargado de abordar la reflexión sobre los modos de encuentro corporal y las políticas de acceso a los espacios de aparición como parte central de la lucha y la reflexión política. Partiendo de la eclosión de movilización ciudadana del 15M (Pérez Navarro, 2014) y la primavera árabe, el autor se centra en los modos de producción del espacio de coalición transfeminista y queer a partir de experiencias activistas recientes en la ciudad de Madrid. En relación, Pérez identifica la segregación de los espacios de la ciudad neoliberal como uno de los principales límites a la hora de interconectar resistencias dentro de los feminismos.

Así pues, los espacios del activismo determinan mediante sus propias dinámicas las posibilidades de apertura hacia otros movimientos. Las posibilidades de la disidencia dependen, según Pérez, de la capacidad para multiplicar puentes y superficies de contacto, para transformar cualquier coalición en aquello que esta todavía no es y para habitar unas políticas comprometidas con su propio desbordamiento crítico. Así, los conflictos o perturbaciones internas en diferentes movimientos son un ejemplo de la capacidad elástica de los mismos por construir alianzas. En este sentido, el autor se centra en el caso de la plataforma Orgullo Crítico (Madrid), donde, a medida que aumenta su complejidad organizativa, van surgiendo perturbaciones críticas que hacen a la propia plataforma replantearse sus objetivos. Son estas tensiones internas las 
que elevan el nivel de autocrítica de los movimientos, constatando que estas tensiones y conflictos en los márgenes no tienen por qué constituir una fuerza centrípeta. Antes bien, anuncian posibilidades de transformación crítica inscritas en las fronteras de cualquier espacio identitario. Y esto nos sugiere oportunidades de enriquecimiento conceptual y práctico para el movimiento feminista en el momento actual.

\section{CONCLUSIÓN}

Las tensiones a las que deben hacer frente los feminismos en la actualidad son muchas y diversas, y gran parte surge a raíz de la torsión que provoca el neoliberalismo en dicho movimiento. Tal y como hemos visto, la gran parte de las tensiones analizadas en este monográfico nos remiten a la dimensión discursiva referida a las lógicas del emprendimiento, el consumo, la autogestión y la productividad, cuestiones que se incorporan y ejercitan tanto por parte de las instituciones como de las subjetividades individuales. Procesos que nos muestran cómo las estructuras de las sociedades neoliberales siguen perpetuando las violencias contra las mujeres y la desigualdad, ahora salvaguardadas tras una aparente sensación de libertad de decisión derivada de un acuciado individualismo.

Sin embargo, una realidad se hace patente: mientras se produce una institucionalización de la búsqueda por la igualdad de género (Reverter-Bañón, 2011) y una creciente popularización del feminismo en la sociedad, las teóricas de la filosofía feminista debemos mostrarnos atentas a los virajes discursivos y las reconceptualizaciones que están sufriendo conceptos otrora claves para la lucha feminista, como son la igualdad, la libertad y la justicia. Los sutiles movimientos discursivos llevados a cabo por el neoliberalismo pueden acabar por cooptar al movimiento por la igualdad, deshaciendo su raíz política característica. En este contexto, las luchas por el reconocimiento y los derechos humanos se convierten en reclamaciones vitales para subvertir las relaciones del dominio patriarcal (Strazzeri, 2007, 2016).

\section{BIBLIOGRAFÍA}

Alario, Mónica (2018). La influencia del imaginario de la pornografía hegemónica en la construcción del deseo sexual masculino 
prostituyente: un análisis de la demanda de prostitución. Asparkía. Investigació Feminista, 33, 13-26. doi: 10.6035/Asparkia.2018.33.4 Banet-Weiser, Sarah (2018). Empowered: Popular Feminism and Popular Misogyny. Durham: Duke University Press.

De Miguel, Ana (2015). Neoliberalismo sexual: el mito de la libre elección. Madrid: Ediciones Cátedra.

Ekman, Kajsa Ekis (2013). Being and being bought: Prostitution, Surrogacy and the Split Self. Victoria: Spinifex Press.

Fraser, Nancy (1997). Heterosexism, Misrecognition, and Capitalism: A Response to Judith Butler. Social Text, 52-53, 279-289.

Fraser, Nancy (2000). Rethinking Recognition: Overcoming Displacement and Reification in Cultural Politics. New Left Review, 3, 107-120.

Fraser, Nancy (2011). Dilemas de la justicia en el siglo XXI. Género y globalización. Palma: UIB.

Fraser, Nancy (2015). Fortunas del feminismo. Quito: IAEN.

Gill, Rosalind (2007). Postfeminist Media Culture Elements of a Sensibility. European Journal of Cultural Studies, 10(2), 147-166. doi: $10.1177 / 1367549407075898$.

Gill, Rosalind (2008). Empowerment/Sexism: Figuring Female Sexual Agency in Contemporary Advertising. Feminism \& Psychology, 18(1), 35-6o. doi: 10.1177/0959353507084950.

Gill, Rosalind (2019). Cuando la propia vida es el campo laboral. Aspectos clave en la gestión de la vida en los empleos vinculados con tecnologías digitales. Recerca. Revista de Pensament $i$ Análisi, 24(1), 14-36. doi: 10.6035/Recerca.2019.24.1.2

Gimeno, Beatriz (2018). La nueva utilidad de la prostitución en el neoliberalismo. Atlántidas. Revista Internacional de Estudios Feministas, 3(1), 13-32.

Hakim, Catherine (2011). Honey Money. The power of erotic capital. London: Allen Lane.

Laval, Christian y Dardot, Pierre (2018). El ser neoliberal. Barcelona: Gedisa. 
Mackie, Vera (1 de agosto, 2016). Closing the gender gap in Japan East Asia Forum -Economics, Politics and Public Policy in East Asia and the Pacific. Recuperado de: http://www.eastasiaforum.org/2016/o8/o1/closing-the-gender-gapin-japan/ [Consultado el 27 de enero de 2017]

Maseda García, Rebeca y Gómez Nicolau, Emma (2018). Time's Up, celebrities and the transformation of gender violence paradigms: The case of Oprah Winfrey's Speech at the Golden Globes (2018). Teknokultura, 15(2): 193-205. doi: 10.5209/TEKN.59362.

McRobbie, Angela (2004). Post-Feminism and Popular Culture. Feminist Media Studies, 4(3), 255-264. doi: 10.1080/1468077042000309937.

McRobbie, Angela (2009). The Aftermath of Feminism: Gender, Culture and Social Change. London: SAGE.

Medina-Vicent, María (2018a). Feminisme neoliberal: un oxímoron? Quaderns de Filosofía, 2, 75-101.

Medina-Vicent, María (2018b). Flirting with Neoliberalism: The Transfiguration of Feminist Political Awareness. NORA. Nordic Journal of Feminist and Gender Research, 26(1), 69-75.

Medina-Vicent, María (2018c). Mujeres y emprendimiento a través de Lean In: una perspectiva crítica. Cuadernos de Relaciones Laborales, 36(2), 305-323.

Medina-Vicent, Maria (2018d). Cuerpos y mercado en la era de la precariedad. Asparkía. Investigació Feminista, 33, 13-26. doi: 10.6035/Asparkia.2018.33.1

Menéndez, María Isabel (2015). Alianzas conceptuales entre patriarcado y postfeminismo: a propósito del capital erótico. Clepsydra, 13, 45-64.

Menéndez, María Isabel (2017). Entre neomachismo y retrosexismo: antifeminismo en industrias culturales. Prisma Social, 2, 1-3o.

Moreno Pestaña, José Luis (2016). La cara oscura del capital erótico. Capitalización del cuerpo y trastornos alimentarios. Madrid: Akal. 
Muñoz-Rodríguez, David y Santos Ortega, Antonio (2017). Las cárceles del capital humano: trabajo y vidas precarias en la juventud universitaria. Recerca. Revista de Pensament i Análisi, 2o, 59-78.

Pérez Navarro, Pablo (2014). Queer Politics of Space in the 15-M Movement. Lambda Nordica, 19(2), 83-114.

Projansky, Sarah (2001). Watching Rape: Film and Television in Postfeminist Culture. New York: New York University Press.

Ranea, Beatriz (2017). (Re)pensar la prostitución desde el análisis crítico de la masculinidad. En Nuño, Laura y de Miguel, Ana (Eds.). Elementos para una teoría crítica del sistema prostitucional (135142). Granada: Comares.

Reverter-Bañón, Sonia (2001). Feminismo y democracia: una crítica antifundamentalista. Recerca. Revista de Pensament $i$ Análisi, 1, 95108.

Reverter-Bañón, Sonia (2009). El ruido de la teoría feminista. Cuadernos Koré, 1, 53-68.

Reverter-Bañón, Sonia (2011). Los retos del feminismo institucional. Daimon. Revista Internacional de Filosofía, suplemento 4, 223-229.

Reverter-Bañón, Sonia (2017). Cosmopolitismo feminista contra globalización. Araucaria, 19, 301-325.

Rottenberg, Catherine (2018a). Women who work: the limits of the neoliberal feminist paradigm. Gender, Work \& Organization, 1-10.

Rottenberg, Catherine (2018b). The Rise of Neoliberal Feminism. Oxford: Oxford University Press.

Sambade, Iván (2017). La instrumentalización de la sexualidad. Masculinidad patriarcal, pornografía y prostitución. En Nuño, Laura y de Miguel, Ana (Eds.). Elementos para una teoría crítica del sistema prostitucional (169-180). Granada: Comares.

Scharff, Christina (2016a). The Psychic Life of Neoliberalism: Mapping the Contours of Entrepreneurial Subjectivity. Theory, Culture \& Society, 33(6), 107-122. 
Scharff, Christina (2016b). Gender and Neoliberalism. Young Women as Ideal Neoliberal Subjects. En Springer, Simon, Birch, Kean y MacLeavy, Julie (Eds.). The Handbook of Neoliberalism (217-226). New York: Routledge.

Schild, Verónica (2016). Feminismo y neoliberalismo en América Latina. Nueva Sociedad, 265, 32-49.

Serrano, Amparo, Fernández Rodríguez, Carlos J. y Artiaga, Alba (2012). Ingenierías de la subjetividad: el caso de la orientación para el desempleo. Revista Española de Investigaciones Sociológicas, 138, 41-62.

Strazzeri, Irene (2007). Riconoscimento e diritti umani. Grammatica del conflitto nel processo di integrazione europea. Perugia: Morlacchi Editore.

Strazzeri, Irene (2016). La resistenza della differenza. Tra liberazione e dominio. Sesto San Giovanni (MI): Mimesis Edizioni.

Strazzeri, Irene (2019). Femminismo liquido. Dalle origini al cyborg. Bari: Progedit.

Tasker, Yvonne y Negra, Diane (2007). Interrogating Postfeminism. Gender and the Politics of Popular Culture. Durham and London: Duke University Press. 
14 RECERCA · DOI: http://dx.doi.org/10.6035/Recerca.2019.24.2.1 - ISSN: 113o-6149-pp. 1-14 